\title{
Correction to Andoh-Arthur, Knizek, Osafo, and Hjelmeland (2019)
}

In the article entitled "Societal reactions to suicide in Ghana: A qualitative study of experiences of the bereaved" by J. Andoh-Arthur, B. L. Knizek, J. Osafo, and H. Hjelmeland (Crisis: Journal of Crisis Intervention and Suicide Prevention, https:/doi.org/10.1027/0227-5910/ a000618) there was an error in Table 1 . The total number of interviewed friends was 16 . However, the total number of friends in the religious affiliations sub-sample came to 17. Instead of 16 Christians it should be 15 Christians.

Thus, the correct table reads as follows (correction in bold):
The authors regret any inconvenience or confusion this computational error may have caused.

\section{Reference}
Andoh-Arthur, J., Knizek, B. L., Osafo, J. \& Hjelmeland, H. (2019). Societal reactions to suicide in Ghana: a qualitative study of ex- periences of the bereaved. Crisis: Journal of Crisis Intervention and Suicide Prevention. Advance online publication. https://doi. org/10.1027/0227-5910/a000618

Published online September 12, 2019

Table 1. Sociodemographic information on participants

\begin{tabular}{|c|c|c|c|c|c|c|c|}
\hline Relation & Male & Female & Total & Age range & Religion & Ethnicity & Education \\
\hline Parents & 1 & 2 & 3 & $50-80$ & All Christians & 3 Akans & $\begin{array}{l}1 \text { Tertiary } \\
2 \text { Elementary }\end{array}$ \\
\hline Spouses & & 6 & 6 & $24-50$ & All Christians & $\begin{array}{l}3 \text { Akans } \\
3 \text { GaDangbes }\end{array}$ & $\begin{array}{l}1 \text { Tertiary } \\
5 \text { Elementary }\end{array}$ \\
\hline Siblings & 8 & 2 & 10 & $28-53$ & All Christians & $\begin{array}{l}7 \text { Akans } \\
1 \text { Ewe } \\
2 \text { Gadangbes }\end{array}$ & $\begin{array}{l}3 \text { Tertiary } \\
7 \text { Elementary }\end{array}$ \\
\hline Friends & 13 & 3 & 16 & $22-59$ & $\begin{array}{l}15 \text { Christians } \\
1 \text { Moslem }\end{array}$ & $\begin{array}{l}9 \text { Akans } \\
1 \text { Ewe } \\
2 \text { Dagomba } \\
3 \text { GaDangbe } \\
1 \text { Grusi }\end{array}$ & $\begin{array}{l}3 \text { Tertiary } \\
11 \text { Elementary } \\
2 \text { No school }\end{array}$ \\
\hline Family relation & 3 & 7 & 10 & $25-70$ & All Christians & $\begin{array}{l}3 \text { Ewes } \\
4 \text { GaDangbes } \\
3 \text { Akans }\end{array}$ & $\begin{array}{l}1 \text { Tertiary } \\
8 \text { Elementary } \\
1 \text { No school }\end{array}$ \\
\hline Total & 25 & 20 & 45 & & & & \\
\hline
\end{tabular}

